\title{
A Spectrograph for the T.60 at Pic du Midi
}

\section{Daniel Bardin and Didier Buty}

Villa Saint-Clair, Chemin de la Marlene, La Pomme, F-13011 Marseille, France

For several years now the 60-cm telescope at Pic du Midi has been made available to amateur astronomers. We felt that this telescope, which fulfils its role of gatherering light very well, could be fitted with equipment to analyze light. To this end the members of the Teachers and Astronomers Liaison Committee (which includes one of the authors of this communication) collaborated in producing and perfecting a spectrograph suitable for the T.60.

Of basic construction, this equipment enables spectra to be recorded on photographic cassettes. It should be transported to the summit at the end of 1987, and will then be available for any amateur projects that may want to use it.

With its focal length of $2100 \mathrm{~mm}$, the T.60 therefore has a focal ratio of 3.5. In imaging the primary image onto the slit, it needed a collimating lens of $f / 3.5$, with an exit pupil appropriate to the area of the grating. In the same way, the field lens of the camera objective needed to receive all the light that had passed through the system.

The components of the spectrograph are as follows, where the numbers refer to the Figure.

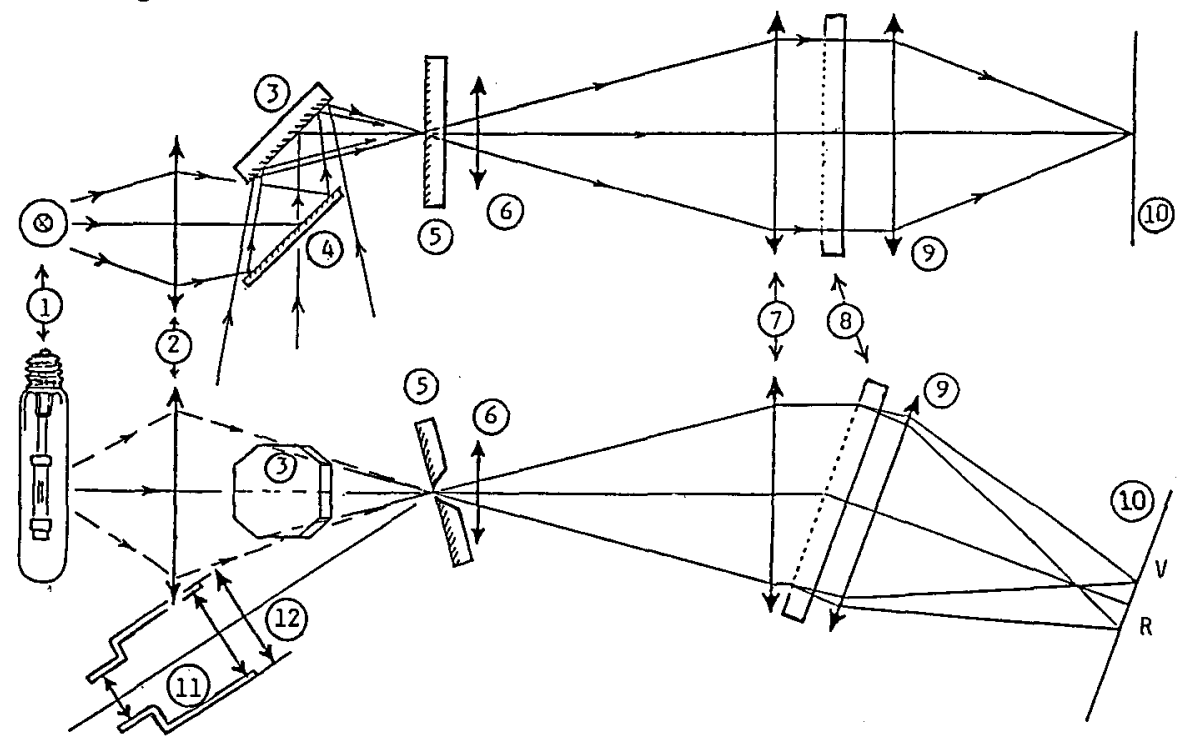


1) argon lamp (reference source); 2) lens focussing light from the argon lamp onto the slit and ensuring that the collimator is fully covered; 3 ) tilting mirror that enables the slit to be swept (in RA), and thus broadens the spectra; 4) mirror that is only used when the reference spectrum is taken; 5) reflecting slit, the reflecting surfaces of which return an image of the field to the small guiding telescope; 6) field lens; 7) collimator; 8) diffraction grating; 9) camera lens; 10) film; 11) centring and guiding telescope; 12) collimator for this telescope.

As far as cameras are concerned, at present these are standard bodies and lenses brought up for each individual amateur project. For example, a lens of $200 \mathrm{~mm}$ aperture, $\mathrm{f} / 3.5$, is a good choice and covers a spectral range of 275 nanometres on a $24 \times 36 \mathrm{~mm}$ frame. A lens of this type was used in the commissioning tests and allows a resolution better than $0.1 \mathrm{~nm}$ in wavelength.

Mounted on the T.60 and in the form it has now, the equipment has already been used (and will be used in future) for various projects, among which we may mention:

1) tests of spectral resolution and sensitometry; comparative tests of gratings, blackand-white and colour emulsions

2) classification of spectra

3) spectral shifts and radial velocities

4) spectral variability: variable stars, emission lines, reinforcement or weakening of lines, abundance problems, stars with high energy losses, spectroscopic doubles, etc.

Undoubtedly this spectrograph is not perfect, it could even have been designed differently. Being suitable for the T.60, however, it should allow amateurs to undertake work in the field of spectroscopy that is comparatively unexplored by them as yet, and at a remarkable site: the Pic du Midi.

The author would like to thank all his colleagues and friends who have accompanied him on various trips, and whose assistance has enabled the project to brought to a successful conclusion. Our thanks are also due to the members of the T.60 Association, to the allocations committee and to all the staff at the Observatory. 